\title{
Corrigendum: The Main Dimensions of Sport Personality Traits: A Lexical Approach
}

\section{OPEN ACCESS}

Edited and reviewed by: Maurizio Bertollo,

G. d'Annunzio University of

Chieti-Pescara, Italy

*Correspondence:

Reinout E. De Vries re.de.vries@vu.n

Specialty section: This article was submitted to Movement Science and Sport

Psychology,

a section of the journal

Frontiers in Psychology

Received: 11 February 2021 Accepted: 30 March 2021

Published: 13 May 2021

Citation:

De Vries RE (2021) Corrigendum: The Main Dimensions of Sport Personality

Traits: A Lexical Approach.

Front. Psychol. 12:666906.

doi: 10.3389/fpsyg.2021.666906

\section{Reinout E. De Vries*}

Department of Experimental and Applied Psychology/Institute of Brain and Behavior Amsterdam, Vrije Universiteit

Amsterdam, Amsterdam, Netherlands

\begin{abstract}
Keywords: sport personality traits, lexical study, HEXACO, Big Five, sport and leisure activities
\end{abstract}
\section{A Corrigendum on}

The Main Dimensions of Sport Personality Traits: A Lexical Approach

by De Vries, R. E. (2020). Front. Psychol. 11:2211. doi: 10.3389/fpsyg.2020.02211

In the original article, there were errors in Tables $\mathbf{4}$ and $\mathbf{5}$ (and in Tables S4.1 through S4.6 in the Supplemental File) as published. Instead of using the natural logarithm (LN) to transform the odd ratios into Cohen's $d$, the common logarithm (LOG) was used. Consequently, all estimates of Cohen's $d$ in Tables $\mathbf{4}$ and $\mathbf{5}$ (and Supplemental Tables S4.1 through S4.6) are underestimates of the true values. The corrected Tables $\mathbf{4}$ and $\mathbf{5}$ and the Supplementary Tables S4.1 through S4.6 to the original article has been updated.

The author apologizes for these errors and states that they do not change the scientific conclusions of the article in any way. The original article has been updated.

Copyright (C) 2021 De Vries. This is an open-access article distributed under the terms of the Creative Commons Attribution License (CC BY). The use, distribution or reproduction in other forums is permitted, provided the original author(s) and the copyright owner(s) are credited and that the original publication in this journal is cited, in accordance with accepted academic practice. No use, distribution or reproduction is permitted which does not comply with these terms. 
TABLE 4 | Logistic regression of the most practiced sports and leisure activities on background and sport personality traits $(N=555)$.

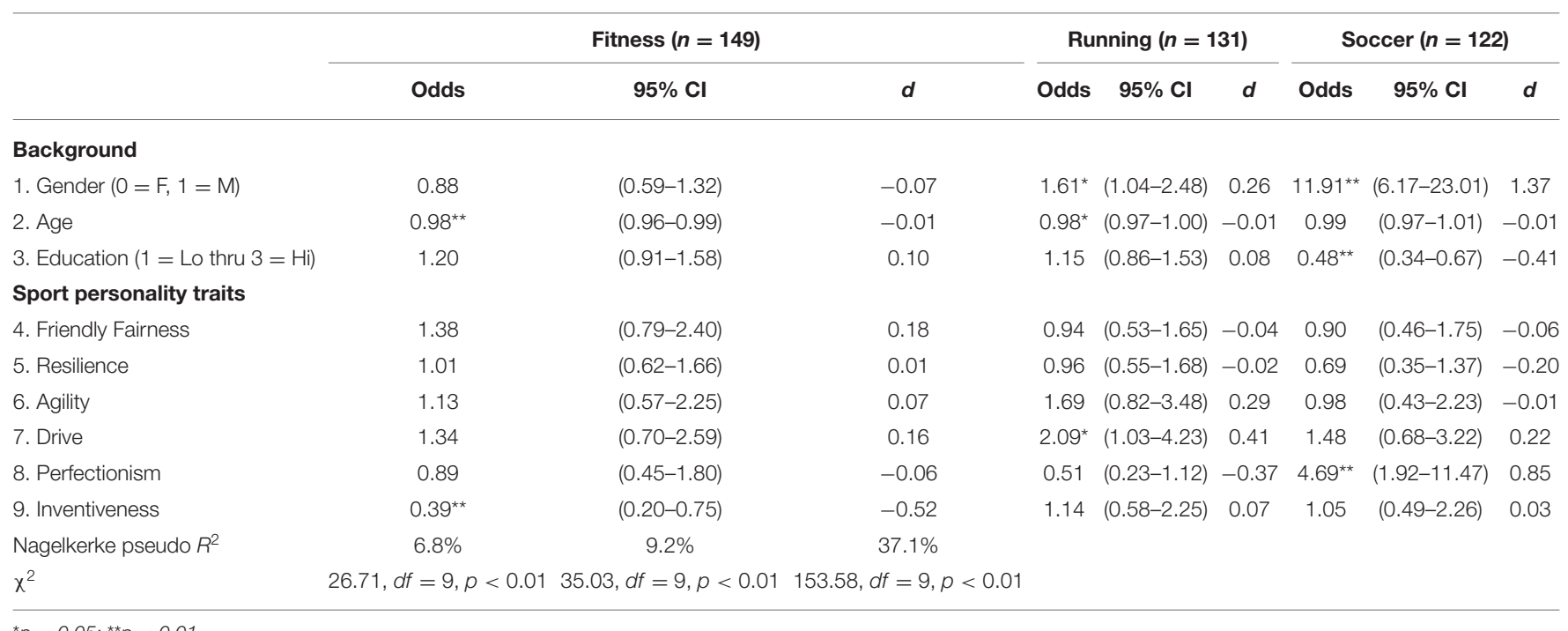

${ }^{*} p<0.05 ;{ }^{* *} p<0.01$

TABLE 5 | Logistic regression of the most practiced sports and leisure activities on background and sport personality traits $(N=555)$.

\begin{tabular}{|c|c|c|c|c|c|c|c|c|c|}
\hline & \multicolumn{3}{|c|}{ Solving puzzles $(n=118)$} & \multicolumn{3}{|c|}{ Swimming $(n=103)$} & \multicolumn{3}{|c|}{ Tennis $(n=98)$} \\
\hline 1. Gender $(0=F, 1=M)$ & $0.32^{\star \star}$ & $(0.20-0.52)$ & -0.62 & $0.26^{\star \star}$ & $(0.16-0.43)$ & -0.75 & 0.72 & $(0.45-1.16)$ & -0.18 \\
\hline 2. Age & $1.07^{\star \star}$ & $(1.05-1.09)$ & 0.04 & 1.00 & $(0.98-1.02)$ & 0.00 & 1.01 & $(1.00-1.03)$ & 0.01 \\
\hline 3. Education $(1=\mathrm{Lo}$ thru $3=\mathrm{Hi})$ & 0.96 & $(0.71-1.31)$ & -0.02 & 1.08 & $(0.79-1.48)$ & 0.04 & $1.43^{\star}$ & (1.03-1.98) & 0.20 \\
\hline 4. Friendly Fairness & 1.12 & $(0.58-2.15)$ & 0.06 & 1.45 & $(0.76-2.76)$ & 0.20 & 1.43 & $(0.75-2.74)$ & 0.20 \\
\hline 5. Resilience & 0.84 & $(0.48-1.46)$ & -0.10 & 1.10 & $(0.63-1.93)$ & 0.05 & 0.61 & $(0.32-1.16)$ & -0.28 \\
\hline 6. Agility & 1.02 & $(0.46-2.26)$ & 0.01 & 0.91 & $(0.41-2.00)$ & -0.05 & $4.05^{\star \star}$ & $(1.74-9.42)$ & 0.77 \\
\hline 7. Drive & 0.66 & $(0.30-1.43)$ & -0.23 & 0.91 & $(0.42-2.01)$ & -0.05 & 0.72 & $(0.32-1.63)$ & -0.18 \\
\hline 8. Perfectionism & 1.58 & (0.69-3.60) & 0.25 & 0.80 & $(0.35-1.82)$ & -0.12 & 1.50 & $(0.63-3.55)$ & 0.22 \\
\hline 9. Inventiveness & 0.82 & $(0.38-1.75)$ & -0.11 & 1.40 & $(0.67-2.93)$ & 0.19 & 0.86 & $(0.41-1.83)$ & -0.08 \\
\hline
\end{tabular}

${ }^{*} p<0.05 ;{ }^{* \star} p<0.01$. 
TABLE S4.1 | Logistic regression of practicing fitness on background, HEXACO personality, and sport personality trait variables $(N=449)$.

\begin{tabular}{|c|c|c|c|c|c|c|}
\hline & \multicolumn{5}{|c|}{ Fitness $(n=125)$} & $D$ \\
\hline 1. $\operatorname{Gender}(0=F, 1=M)$ & 0.78 & $(0.48-1.26)$ & -0.14 & 0.79 & $(0.48-1.30)$ & -0.13 \\
\hline 2. Age & $0.98^{\star}$ & $(0.97-1.00)$ & -0.01 & $0.98^{*}$ & $(0.96-1.00)$ & -0.01 \\
\hline 3. Education $(1=\mathrm{Lo}$ thru $3=\mathrm{Hi})$ & 1.22 & $(0.89-1.69)$ & 0.11 & 1.22 & $(0.88-1.70)$ & 0.11 \\
\hline 4. Honesty-Humility & 1.43 & $(0.84-2.42)$ & 0.20 & 1.21 & $(0.68-2.13)$ & 0.11 \\
\hline 5. Emotionality & 1.15 & $(0.66-1.98)$ & 0.08 & 1.05 & $(0.60-1.85)$ & 0.03 \\
\hline 6. Extraversion & 1.17 & $(0.71-1.94)$ & 0.09 & 1.20 & $(0.69-2.10)$ & 0.10 \\
\hline 7. Agreeableness & 0.75 & $(0.43-1.31)$ & -0.16 & 0.81 & $(0.46-1.43)$ & -0.12 \\
\hline 8. Conscientiousness & 0.93 & $(0.52-1.68)$ & -0.04 & 0.84 & $(0.45-1.59)$ & -0.10 \\
\hline 9. Openness to Experience & 0.92 & $(0.56-1.52)$ & -0.05 & 1.10 & $(0.64-1.88)$ & 0.05 \\
\hline 12. Agility & & & & 1.32 & $(0.60-2.90)$ & 0.15 \\
\hline 13. Drive & & & & 1.45 & $(0.69-3.07)$ & 0.20 \\
\hline 14. Perfectionism & & & & 0.92 & (0.40-2.09) & -0.05 \\
\hline 15. Inventiveness & & & & $0.32^{* *}$ & $(0.15-0.70)$ & -0.63 \\
\hline Nagelkerke pseudo $R^{2}$ & $4.7 \%$ & $8.1 \%$ & & & & \\
\hline$x^{2}$ & $14.75, d f=9, p=0.10$ & $25.97, d f=15, p=0.04$ & & & & \\
\hline
\end{tabular}

TABLE S4.2 | Logistic regression of practicing running on background, HEXACO personality, and sport personality trait variables $(N=449)$.

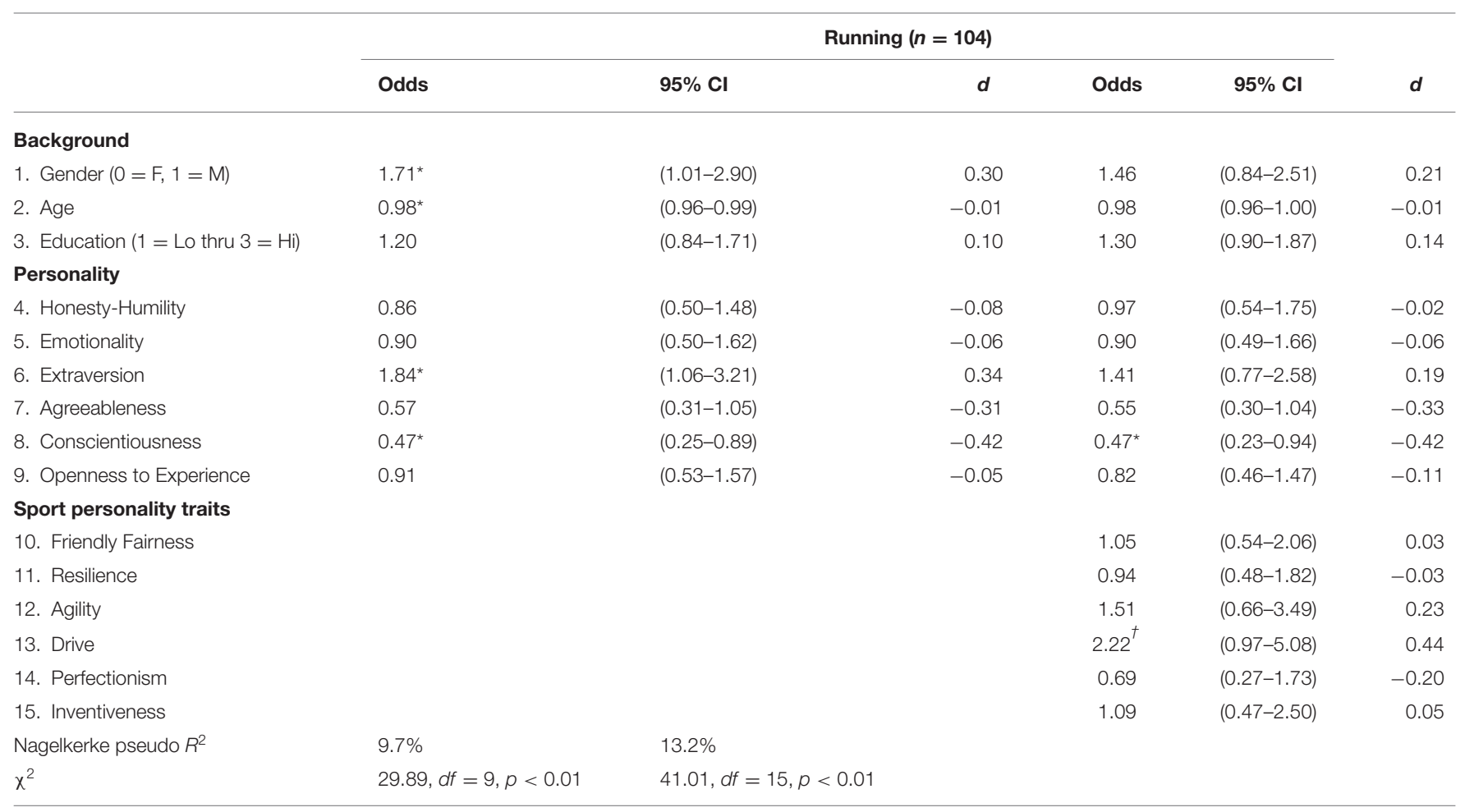

${ }^{*} p<0.05 ;{ }^{* *} p<0.01 ;{ }^{\dagger} p=0.059$ 
TABLE S4.3 | Logistic regression of playing soccer on background, HEXACO personality, and sport personality trait variables $(N=449)$.

\begin{tabular}{|c|c|c|c|c|c|c|}
\hline & \multicolumn{5}{|c|}{ Soccer $(n=93)$} & $\boldsymbol{D}$ \\
\hline \multicolumn{7}{|l|}{ Background } \\
\hline 1. $\operatorname{Gender}(0=F, 1=M)$ & $15.54^{\star \star}$ & $(6.77-35.66)$ & 1.51 & $15.07^{\star \star}$ & $(6.30-36.04)$ & 1.50 \\
\hline 2. Age & 0.99 & $(0.97-1.01)$ & -0.01 & 1.00 & $(0.98-1.02)$ & 0.00 \\
\hline 3. Education $(1=\mathrm{Lo}$ thru $3=\mathrm{Hi})$ & $0.52^{\star \star}$ & $(0.35-0.77)$ & -0.36 & $0.49^{\star \star}$ & $(0.32-0.75)$ & -0.39 \\
\hline 4. Honesty-Humility & 0.56 & $(0.30-1.05)$ & -0.32 & 0.66 & $(0.33-1.35)$ & -0.23 \\
\hline 5. Emotionality & 1.04 & $(0.53-2.06)$ & 0.02 & 1.08 & $(0.52-2.24)$ & 0.04 \\
\hline 6. Extraversion & 1.12 & $(0.59-2.14)$ & 0.06 & 1.17 & $(0.55-2.49)$ & 0.09 \\
\hline 7. Agreeableness & 1.39 & $(0.71-2.71)$ & 0.18 & 1.37 & $(0.67-2.79)$ & 0.17 \\
\hline 8. Conscientiousness & 1.42 & $(0.66-3.03)$ & 0.19 & 0.86 & $(0.38-1.98)$ & -0.08 \\
\hline 9. Openness to Experience & 0.64 & $(0.34-1.22)$ & -0.25 & $0.47^{*}$ & $(0.23-0.96)$ & -0.42 \\
\hline 12. Agility & & & & 1.02 & $(0.39-2.71)$ & 0.01 \\
\hline 13. Drive & & & & 0.92 & $(0.34-2.45)$ & -0.05 \\
\hline 14. Perfectionism & & & & $9.16^{\star \star}$ & $(3.05-27.47)$ & 1.22 \\
\hline 15. Inventiveness & & & & 0.68 & $(0.26-1.79)$ & -0.21 \\
\hline Nagelkerke pseudo $R^{2}$ & $32.7 \%$ & $39.8 \%$ & & & & \\
\hline$x^{2}$ & $105.38, d f=9, p<0.01$ & $131.73, d f=15, p<0.01$ & & & & \\
\hline
\end{tabular}

TABLE S4.4 | Logistic regression of solving puzzles on background, HEXACO personality, and sport personality trait variables $(N=449)$.

\begin{tabular}{|c|c|c|c|c|c|c|}
\hline & \multicolumn{5}{|c|}{ Solving Puzzles $(n=98)$} & \multirow[b]{2}{*}{$d$} \\
\hline & Odds & $95 \% \mathrm{Cl}$ & $d$ & Odds & $95 \% \mathrm{Cl}$ & \\
\hline \multicolumn{7}{|l|}{ Background } \\
\hline 1. Gender $(0=F, 1=M)$ & $0.32^{* \star}$ & $(0.18-0.58)$ & -0.63 & $0.33^{\star *}$ & $(0.18-0.59)$ & -0.61 \\
\hline 2. Age & $1.06^{\star \star}$ & $(1.04-1.09)$ & 0.03 & $1.06^{\star \star}$ & $(1.04-1.09)$ & 0.03 \\
\hline 3. Education $(1=$ Lo thru $3=\mathrm{Hi})$ & 0.92 & $(0.65-1.31)$ & -0.05 & 0.90 & $(0.63-1.29)$ & -0.06 \\
\hline 4. Honesty-Humility & 1.07 & $(0.56-2.04)$ & 0.04 & 1.03 & $(0.52-2.06)$ & 0.02 \\
\hline 5. Emotionality & 0.95 & $(0.49-1.84)$ & -0.03 & 0.95 & $(0.49-1.87)$ & -0.03 \\
\hline 6. Extraversion & $0.39^{\star *}$ & $(0.21-0.71)$ & -0.52 & $0.41^{\star \star}$ & $(0.21-0.81)$ & -0.49 \\
\hline 7. Agreeableness & 1.57 & (0.81-3.03) & 0.25 & 1.55 & $(0.79-3.05)$ & 0.24 \\
\hline 8. Conscientiousness & 1.44 & $(0.71-2.93)$ & 0.20 & 1.35 & $(0.64-2.85)$ & 0.17 \\
\hline 12. Agility & & & & 0.77 & $(0.32-1.87)$ & -0.14 \\
\hline 13. Drive & & & & 0.92 & $(0.39-2.19)$ & -0.05 \\
\hline 14. Perfectionism & & & & 1.35 & $(0.51-3.57)$ & 0.17 \\
\hline 15. Inventiveness & & & & 1.02 & $(0.42-2.47)$ & 0.01 \\
\hline Nagelkerke pseudo $R^{2}$ & $22.7 \%$ & $23.0 \%$ & & & & \\
\hline$x^{2}$ & $71.80, d f=9, p<0.01$ & $72.53, d f=15, p<0.01$ & & & & \\
\hline
\end{tabular}


TABLE S4.5 | Logistic regression of practicing swimming on background, HEXACO personality, and sport personality trait variables $(N=449)$.

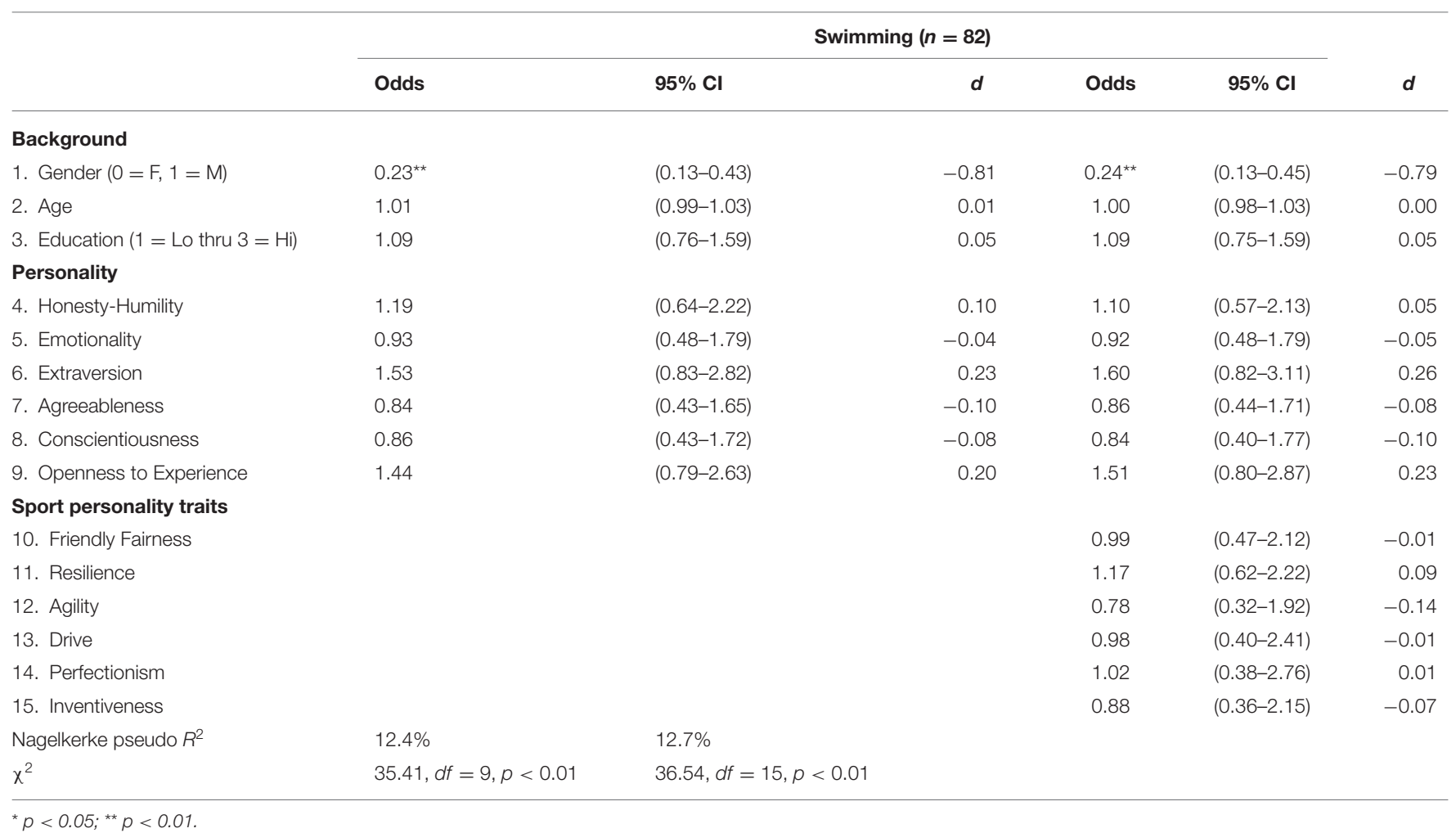

TABLE S4.6 | Logistic regression of playing tennis on background, HEXACO personality, and sport personality trait variables $(N=449)$.

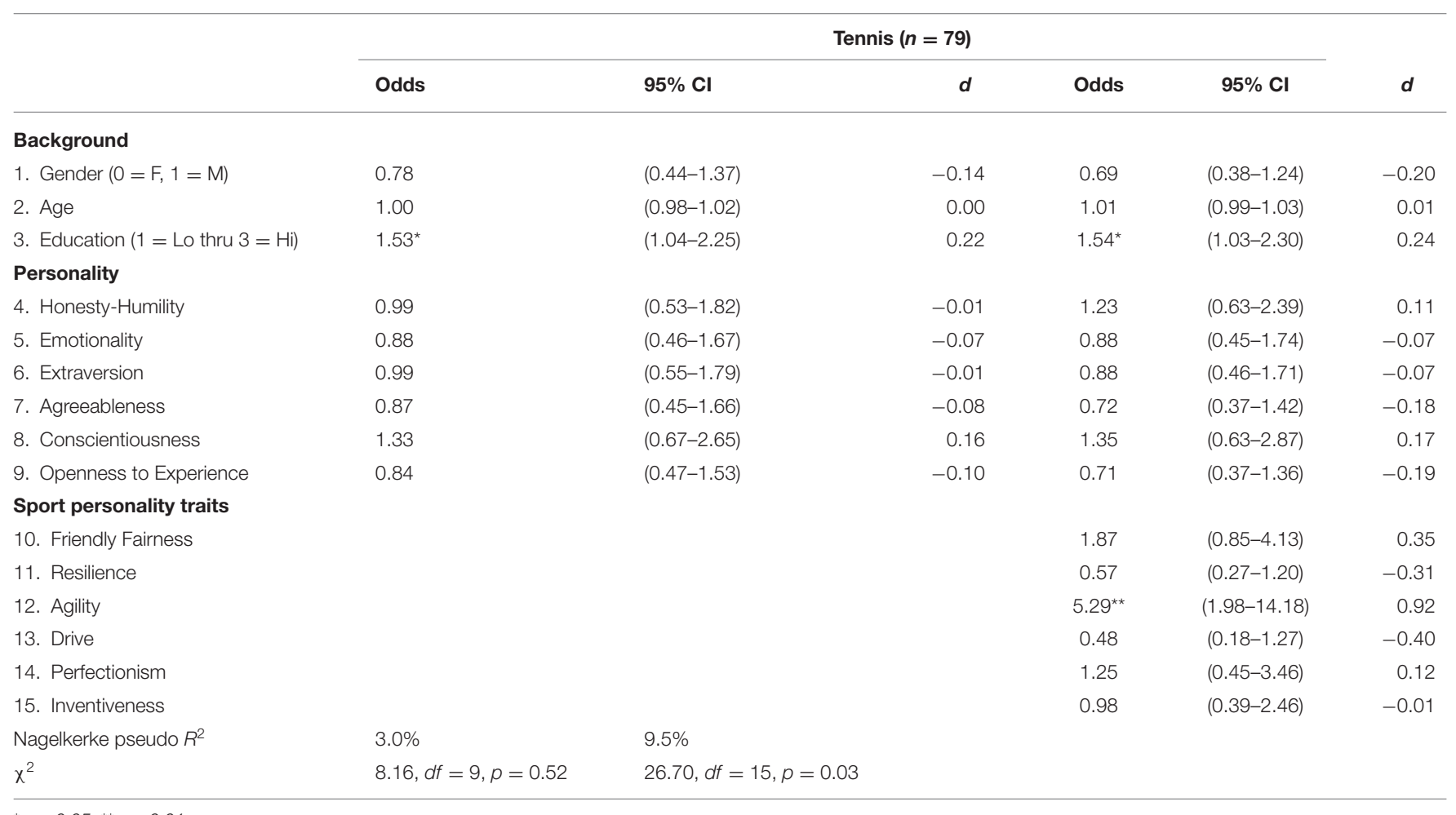

